\title{
The 'yielding transition' in crystal plasticity - discrete dislocations and continuum models
}

\author{
Michael Zaiser ${ }^{* \dagger}$ \\ CNR, Istituto dei Sistemi Complessi, Via dei Taurini 19, 00185 Roma, Italy \\ E-mail: M. Zaiser@ed.ac.uk
}

\section{Brett Marmo and Paolo Moretti}

The University of Edinburgh, Institute for Materials and Processes

The King's Buildings, Sanderson Building, Edinburgh EH93JL, UK

\begin{abstract}
On mesoscopic and microscopic scales, plastic deformation of crystalline solids is characterized by large intrinsic spatio-temporal fluctuations with scale-invariant characteristics: In time, deformation proceeds through intermittent bursts with power-law size distributions; in space, deformation patterns and deformation-induced surface morphology are characterized by long-range correlations, self-similarity and/or self-affine roughness. We discuss this scale-invariant behavior in terms of robust scaling associated with a non-equilibrium critical point ('yielding transition'). The properties of this transition are studied using two conceptually different models which, however, are shown to yield qualitatively and quantitatively similar results.
\end{abstract}

International Conference on Statistical Mechanics of Plasticity and Related Instabilities

31 August - 2 September 2005

Bangalore, India

\footnotetext{
* Speaker.

$\dagger$ Permanent address: The University of Edinburgh, Institute for Materials and Processes, The King's Buildings, Sanderson Building, Edinburgh EH93JL, UK
} 


\section{Introduction}

In recent years, the traditional paradigm of 'stable' plastic deformation as a smooth and laminar flow process has been challenged both experimentally and theoretically. Experimentally, acoustic emission studies reveal large intermittent temporal fluctuations of the deformation rate [1]. It is observed that the loci of plastic activity are distributed in space according to fractal patterns [2], and that the surface topography induced by plastic deformation is characterized by self-affine scaling properties [3, 4]. In summary, plastic deformation is characterized by huge intrinsic fluctuations which exhibit scale-free patterns in space and in time. Such fluctuations become directly observable in the deformation of micron-scale samples where they manifest themselves through steps in the stress-strain curves and huge scatter in the flow stresses of samples with identical process history $[5,6]$.

In order to give a theoretical interpretation of these fluctuation phenomena, several authors have invoked the concept of 'self-organized criticality' $[2,5]$ though it is hardly ever specified what this should mean in the context of plasticity. Others have made the proposal that plastic yielding, i.e. the transition of a crystal from an elastically deforming to a plastically flowing state, may be envisaged as a second-order-like non-equilibrium phase transition ('yielding transition') [7, 8]. In this interpretation, scale-free fluctuation phenomena in plastic flow may be associated with the critical behavior near the yield stress.

In the following we are going to follow up this interpretation by studying the 'yielding transition' of two simplified models of plastic flow, using (a) a dislocation-based model which envisages plastic flow in terms of the deterministic evolution of a - rather simplified - dislocation system, and (b) a stochastic continuum model which models heterogeneities in microstructure evolution in terms of a stochastic evolution of the local flow stress. It is important to emphasize that our models do not pretend to provide a fully realistic description of the details of dislocation processes in particular materials. Rather, it is our aim to use very simple models in order to work out key features of the collective processes which occur as a crystal begins to deform plastically. If the basic concept of yielding as a non-equilibrium phase transition with critical behavior is viable, then because of the universality of critical behavior the predictions of such simple models should be in qualitative agreement with the observations made in different types of materials. We will demonstrate in the following that this is indeed the case.

\section{Critical behavior at yield: A dislocation-based model}

As a most simple model of plastic yielding, we consider an ensemble of straight parallel edge dislocations moving on a single slip system. Slip occurs in the $x$ direction, and the system is homogeneous in $z$. We assume equal numbers of positive and negative dislocations, without dislocation multiplication or annihilation. To prevent loss of dislocations through the boundaries of the simulated volume, we impose periodic boundary conditions for dislocation fluxes, and similarly for the dislocation stress fields. The externally applied shear stress $\tau_{\text {ext }}$ is considered space-independent over the region of interest. 


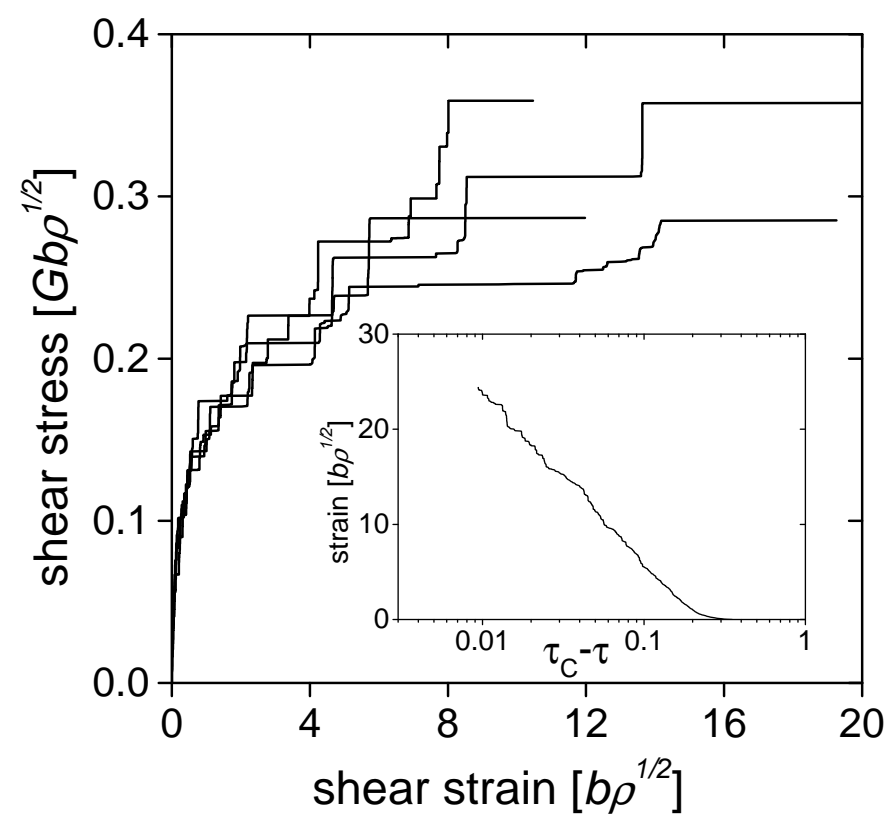

Figure 1: Stress-strain curves in simulated stress-controlled tests on systems of size $32 \times 32 / \rho$ (1024 Dislocations); insert: average stress-strain behavior obtained by averaging over 100 simulations.

The motion of each dislocation occurs under the action of an applied stress, plus the stresses created by all other dislocations. The shear stress acting on the $j$ th dislocation is, hence, given by

$$
\tau\left(\vec{r}_{j}\right)=\tau_{\mathrm{ext}}+\sum_{i \neq j} s_{i} \tau_{\mathrm{D}}\left(\vec{r}_{i}-\vec{r}_{j}\right)
$$

where $\tau_{\mathrm{D}}(\vec{r})$ is the shear stress (xy-component of the stress tensor) created at $\vec{r}$ by a positive edge dislocation located at the origin. Space is discretized into a grid and an automaton-type of dynamics is implemented: A dislocation is moved by a unit amount in the direction imposed by the stress and the sign of the dislocation (a positive dislocation moves in the $+x$ direction under a positive stress). A move is accepted if the sign of the stress acting on the dislocation does not change during the move. All dislocations are moved simultaneously, and then the new stresses are computed (simultaneous update). Alternatively, also an extremal dynamics was implemented where only the dislocation experiencing the largest stress is moved.

Initially, dislocations are placed at random locations. We then carry out a relaxation at zero applied stress until the dislocation system has settled into a stable configuration. Subsequently, an external stress is applied and the evolution of the dislocation system is studied. We consider deformation in a stress-controlled mode where the applied stress is increased from zero, and the stress-strain curve is recorded. The external stress increase is adiabatically slow, i.e., a (small) external stress increment is applied only after all dislocation activity had ceased.

Stress-strain characteristics are determined by simultaneously recording the stress and the total strain $\gamma=\left(b / L_{\mathrm{s}}^{2}\right) \sum_{i} s_{i} L_{i}$, where $L_{\mathrm{s}}^{2}$ is the simulated area, $s_{i}$ is the sign of the $i$ th dislocation and $L_{i}=x_{i}-x_{i, 0}$ is its (if necessary periodically continued) glide path. Simulations are terminated at a prescribed maximum strain. Results are shown in Figure 1. The stress-strain curves assume a 


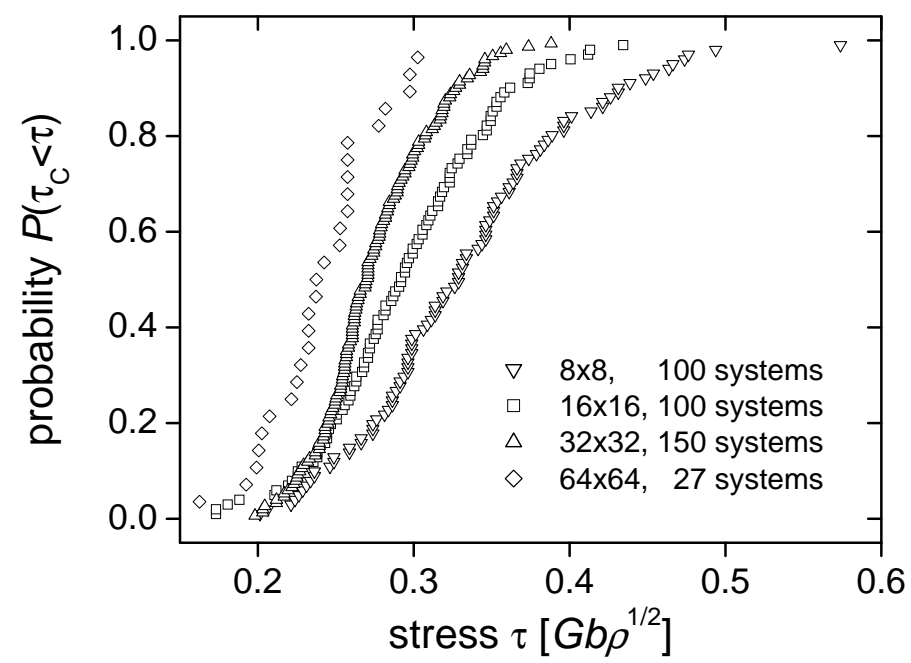

Figure 2: Distributions of yield stresses obtained for systems of different sizes (system size in units of $1 / \sqrt{\rho})$.

staircase-like shape, which is reminiscent of the experimental curves of micron-sized specimens reported by Dimiduk and co-workers [5, 6]. As in the experimental observations, there is substantial scatter between different simulations, which does not represent any differences in the material properties but simply reflects the outcome of different initial positions of the dislocations.

By averaging over many simulations, a smooth stress-strain characteristics can be obtained. By plotting the average stress-strain curve in semi-logarithmic coordinates, it becomes evident that the strain diverges logarithmically as the stress approaches a critical value $\tau_{\mathrm{c}}$. This divergence corresponds to a horizontal asymptote in the stress vs strain graph: The model asymptotically displays ideally plastic behavior, and $\tau_{\mathrm{c}}$ is the yield stress. (The absence of hardening is to be expected, since no dislocation multiplication mechanism was introduced.) As a corollary, we note that the susceptibility $\chi:=\partial \gamma / \partial \tau_{\text {ext }}$ of the plastic strain diverges at the yield stress like $\chi \propto\left(\tau_{\mathrm{c}}-\right.$ $\left.\tau_{\text {ext }}\right)^{-1}$.

The statistical distribution of critical stresses obtained from different simulations of statistically equivalent systems depends on system size. This is illustrated in Figure 2 which shows probability distributions of yield stresses obtained from ensembles of systems of area $L_{\mathrm{S}}^{2}=8 \times 8 / \rho$ to $L_{\mathrm{s}}^{2}=64 \times 64 / \rho$ corresponding, for a typical dislocation density of $\rho=10^{12} \mathrm{~m}^{-1}$, to sizes of $8 \times 8$ to $64 \times 64\left(\mu \mathrm{m}^{2}\right)$. It is seen that the width of the distributions increases with decreasing system size, indicating an increasing scatter in the deformation behavior. At the same time, the average yield stress increases for smaller systems. This size effect is of a purely statistical nature and does not relate to surface effects or effects of dislocation sources, as periodic boundary conditions were used and a conserved number of dislocations was assumed. Both the increasing scatter and the increase in strength with decreasing system size match the observations in deformation of micron-sized specimens reported by Dimiduk et. al. [5, 6].

The behavior in the individual simulations is characterized by large steps in the stress-strain curves (dislocation avalanches). The statistics of these avalanches has been investigated as a 

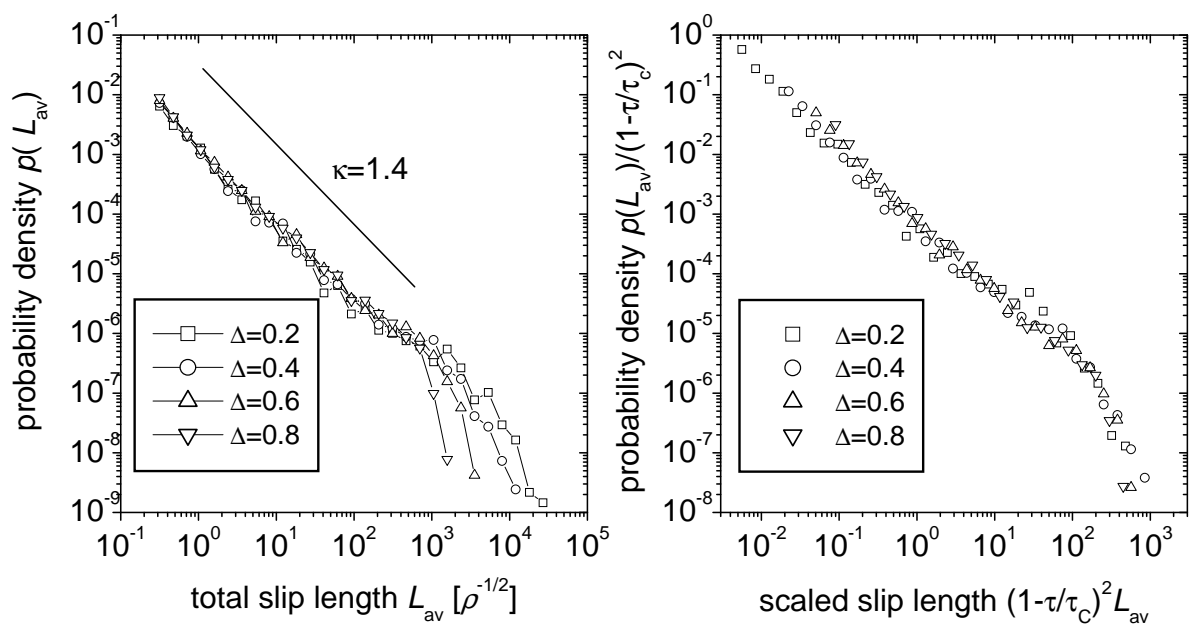

Figure 3: Distributions of dislocation avalanche sizes measured in terms of the total slip distance covered by dislocations during an avalanche; right: collapse of distributions obtained for different sized if size is re-scaled by $L_{\mathrm{av}} \rightarrow L_{\mathrm{av}}\left(1-\tau / \tau_{\mathrm{c}}\right)^{2}$;.

function of stress. Avalanches were characterized in terms of the total slip length increment $L_{\mathrm{av}}=\sum_{i} s_{i} \Delta L_{i}$ where $\Delta L_{i}$ is the difference between the positions of the $i$ th dislocation before and after the avalanche. This makes it possible to directly compare simulations of systems of different area - the total strain increment $\Delta \gamma=b L_{\mathrm{av}} / L_{\mathrm{s}}^{2}$, on the other hand, is inversely proportional to the simulated area $L_{\mathrm{s}}^{2}$. Avalanche size distributions $p_{\tau}\left(L_{\mathrm{av}}\right)$ were determined over narrow stress intervals centered around different stresses $\tau$. Results are shown in Figure 3 for $\Delta=\left(1-\tau_{\text {ext }} / \tau_{\mathrm{c}}\right)=0.2 \ldots 0.8$.

One observes power-law distributions of dislocation avalanche sizes with a common exponent $\kappa \approx 1.4$. Whereas this exponent does not depend on stress, the maximum avalanche size increases like $\left(1-\tau_{\text {ext }} / \tau_{\mathrm{c}}\right)^{-1 / \sigma}$ with $\sigma \approx 0.5$ as the stress approaches the yield stress. Accordingly, distributions pertaining to different stress levels obey the scaling form

$$
p_{\tau}\left(L_{\mathrm{av}}\right)=L_{\mathrm{av}}^{-\kappa} f_{L}\left(\frac{L_{\mathrm{av}}}{\Delta^{1 / \sigma}}\right)
$$

These distributions can therefore be collapsed by re-scaling $L_{\mathrm{av}} \rightarrow L_{\mathrm{av}} \Delta^{1 / \sigma}$ (Figure 3, right). It is also seen that power-law scaling is quite robust as scaling over more than three decades persists even at stresses that are just one fifth of the critical stress. This observation of robust scaling behavior quite far from a critical point has also been made in other systems exhibiting avalanche dynamics [9].

Avalanche size distributions can be directly determined from experiments, since they correspond to distributions of step sizes on the stress-strain graphs of micron-sized samples. However, it may be difficult to determine the stress-dependent distributions $p_{\tau}$ since good statistics requires averaging over a huge number of samples. It is much easier to determine the integral distribution of avalanche sizes along the stress-strain curve, which is related to the stress-dependent distributions via

$$
p_{\text {int }}\left(L_{\mathrm{av}}\right) \propto \int p_{\tau}\left(L_{\mathrm{av}}\right) \mathrm{d} \tau \propto L_{\mathrm{av}}^{-(\kappa+\sigma)} .
$$


This distribution exhibits no cut-off but a modified avalanche exponent, which here is expected to be $\kappa+\sigma \approx 1.9$. This exponent by itself does not characterize critical behavior - rather, it combines two different exponents characterizing (i) the scaling regime of the avalanche size distribution and (ii) the scaling of its cut-off. A value close to 2 for this integral exponent, as it follows from our simulations, is in reasonable agreement with preliminary results obtained from stress-strain curves of micron-sized samples [10].

The behavior of conserved two-dimensional dislocation systems with automaton or extremal dynamics under slow loading can be summarized as follows: (i) The strain diverges logarithmically as the stress approaches a critical value (yield stress); (ii) the response of the system to an increasing stress is characterized by an irregular sequence of strain bursts with power-law size distribution. (ii) This scale-free behavior is manifest already at stresses well below the yield stress and persists up to a maximum burst size which diverges at yield. (iii) The behavior of the system does not depend on details of the dynamics such as the order of updates, as both simulations with parallel update and with extremal dynamics yield practically identical results. (iv) The exponents characterizing the critical behavior (avalanche exponent $\kappa \approx 1.4$, divergence of the maximum burst size like $(\tau-$ $\left.\tau_{\mathrm{c}}\right)^{-2}$, divergence of the susceptibility like $\left(\tau-\tau_{\mathrm{c}}\right)^{-1}$ ) correspond to the mean-field behavior of a depinning elastic manifold [11]. Since the main ingredients of depinning theories are absent from our model (there is neither an elastic manifold in the model, nor quenched disorder), one may ask whether this agreement is purely coincidental. To answer this question, it useful to look at an apparently quite different approach which describes plastic deformation in terms of the evolution of a continuous plastic strain field.

\section{Continuum model of the 'yielding transition}

Our first model directly traces the motion and collective dynamics of dislocations. Randomness, heterogeneity and stochastic behavior stem from the probabilistic choice of initial conditions for the otherwise deterministic evolution of a discrete dislocation system. An alternative approach consists in the adaptation of continuum plasticity models to include microstructural heterogeneity and randomness in a phenomenological manner.

\subsection{Constitutive equations}

Continuum mechanical constitutive equations connect the stress, plastic strain, and strain rate in a material. Such models operate on scales above the 'microscopic' scale where individual dislocations may be resolved. Accordingly, stress and strain are now considered as mesoscopic fields which are space dependent on a scale which is above the dislocation spacing, but small in comparison with the dimensions of the deforming body. Stresses associated with individual dislocations are not resolved, but enter the formulation implicitly since short-range dislocation interactions determine the local yield stress of the material and its evolution with strain.

We formulate our model for the same deformation geometry as in the previous section, viz, plane strain deformation of an isotropic material by slip on a single slip system. The problem of evaluating the stress state of the material can be split into two parts: (i) Tractions applied from outside to the surface of the deforming body, or displacements prescribed on the surface of that body, create an 'external' stress (shear stress $\tau_{\text {ext }}$ ) which we consider space-independent over the 
region of interest. (ii) Because of heterogeneities in the material properties, plastic deformation may in general proceed in a spatially heterogeneous manner on mesoscopic scales. Deformation heterogeneities give rise to eigenstresses $\tau_{\text {int }}$. (Note that the stresses associated with dislocations are just a special case of such eigenstresses; the heterogeneity in this case corresponds to the boundary of a slipped area.) Provided that surface effects can be disregarded over the region of interest, the internal shear stress can be written as a functional of the plastic strain field [12, 13]:

$$
\begin{aligned}
\tau_{\text {int }}(\vec{r}) & =\frac{G}{2 \pi(1-v)} \int \gamma\left(\vec{r}^{\prime}\right)\left[\frac{1}{\left(\vec{r}-\vec{r}^{\prime}\right)^{2}}-\frac{8\left(x-x^{\prime}\right)^{2}\left(y-y^{\prime}\right)^{2}}{\left(\vec{r}-\vec{r}^{\prime}\right)^{6}}\right] \mathrm{d}^{2} \vec{r}^{\prime} \\
& +\frac{G}{4(1-v)}[\langle\gamma\rangle-\gamma(\vec{r})],
\end{aligned}
$$

or, in Fourier space,

$$
\tau_{\text {int }}(\vec{k})=-\frac{G}{\pi(1-v)} \gamma(\vec{k}) \frac{k_{x}^{2} k_{y}^{2}}{|\vec{k}|^{4}} .
$$

Regarding these expressions, two points may be noted for later use: (i) The elastic kernel is not positively definite in real space. (ii) Strain fluctuations with wavevectors parallel to the $x$ or $y$ directions do not give rise to internal stresses.

To relate the stress to the evolution of the strain field $\gamma(\vec{r})$, we adopt a linear viscoplastic constitutive relation which contains a gradient-dependent term (for discussion, see $[12,13]$ ). The evolution of the local shear strain $\gamma$ is given by

$$
\mu \partial_{t} \gamma(\vec{r})=\tau(\vec{r})+D \gamma_{x x}-\delta \tau(\gamma, \vec{r})
$$

Here $\tau=\tau_{\text {ext }}+\tau_{\text {int }}$ is the locally acting shear stress which derives from solving the elastic problem. The other two terms on the right-hand side of this equation represent local stresses on the 'microscopic' scale of individual dislocations which are not resolved in detail on the mesoscopic scale on which the strain field $\gamma$ is defined. The first of these terms arises from short-range interactions of small groups of dislocations gliding collectively on the same or on adjacent slip planes; in this term, $D \approx G / \rho$ and $\rho$ is the dislocation density [14]. The second term represents in a summary manner fluctuations of the internal stress field arising from the interactions of discrete dislocations; this term has the correlation function

$$
\langle\delta \tau\rangle=0, \quad\left\langle\delta \tau(\vec{r}, \gamma) \delta \tau\left(\vec{r}+\vec{r}^{\prime}, \gamma+\gamma^{\prime}\right)\right\rangle=\left\langle\delta \tau^{2}\right\rangle f\left(\vec{r}^{\prime} / \xi, \gamma^{\prime} / \gamma_{\mathrm{c}}\right)
$$

where $\left\langle\delta \tau^{2}\right\rangle \approx G^{2} b^{2} \rho$, the correlation length $\xi \approx 1 / \sqrt{\rho}$ of the fluctuating stress field created by individual dislocations is of the order of the dislocation spacing, and $f$ is a non-dimensional correlation function with $f(0,0)=1$ and characteristic ranges $\xi$ and $\gamma_{\mathrm{c}}$ in its respective arguments. The 'correlation strain' $\gamma_{c} \approx b \sqrt{\rho}$ is estimated as the strain accomplished when all dislocations move by one average dislocation spacing (for details see $[12,13]$ and references therein).

The constitutive model specified by Eqs. (3.1) - (3.4) has the formal structure of models studied in the theory of elastic manifold depinning [11]. Moreover, it is clear from the first term in Eq. (3.1) that the elastic interactions in the present model have mean-field character. Hence, we expect the transition of the model to the 'moving' phase - which corresponds to sustained plastic flow - to be in the universality class of mean-field depinning. 


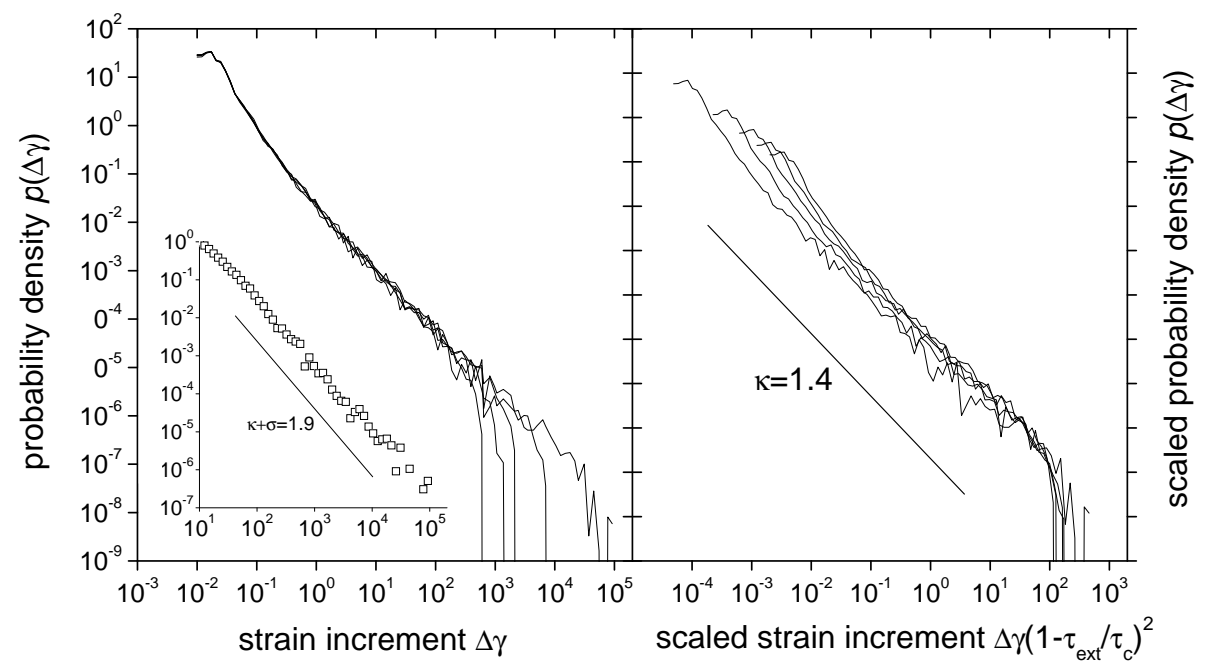

Figure 4: Probability distributions of slip avalanche sizes (probability density $p(\Delta \gamma)$ vs. strain increment $\Delta \gamma)$ as obtained from an ensemble of systems of size $128 \times 128$; left: distributions corresponding to different stresses $\left(\Delta=1-\tau_{\text {ext }} / \tau_{\mathrm{c}}\right)=0.1 \ldots 0.5$; right: scaling collapse after re-scaling $\Delta \gamma \rightarrow \Delta \gamma\left(1-\tau_{\mathrm{ext}} / \tau_{\mathrm{c}}\right)^{2}$; insert: total distribution (only large events with $\Delta \gamma>10$ ).

In the following, we report results obtained from simulations of the continuum model. In these simulations, periodic boundary conditions have been imposed as in the previous section. Again, the stress was increased quasi-statically. In practice this means that a small stress increment was applied whenever the strain rate fell below a small threshold value, and it was checked that the results did not change appreciably [i.e., above the noise level inherent in the simulations] when either the magnitude of the stress increment or the threshold strain rate were further decreased.

\subsection{Avalanche dynamics and surface morphology evolution}

Stress-strain curves and slip avalanches The simulated stress-strain graphs (for examples, see [12]) exhibit the same staircase-like characteristics as those observed in 2D dislocation dynamics simulations and in experiments with micron-sized specimens. Again, the strain diverges at some critical stress $\tau_{\mathrm{c}}$ where the stress-strain curve reaches a horizontal tangent, and the ensembleaveraged susceptibility $\chi=\partial\langle\gamma\rangle / \partial \tau$ of the plastic strain diverges as $\chi \propto\left(\tau_{\mathrm{c}}-\tau\right)^{-1}$.

Close to the critical stress, the stress-strain curves have the structure of a devil's staircase where the step sizes (sizes of slip avalanches, defined as strain increments following a stress increment) obey a scale-free distribution. Figure 4 shows distributions of step sizes $\Delta \gamma$ obtained over narrow stress intervals (width 0.01 in scaled variables) and at various distances from the critical stress. In this and in the following figures, length, stress, and strain are measured in the respective 'natural units' $1 / \sqrt{\rho}, G b \sqrt{\rho}$, and $b \sqrt{\rho}$ for a dislocation system. (No strain increments are shown that are less than $1 / L_{s}^{2}$ in non-dimensional units, since these correspond to local adjustments to a stress increment rather than representing collective behavior.) As one approaches the critical stress, the power-law scaling $p(\Delta \gamma) \propto \Delta \gamma^{-\kappa}$ with $\kappa \approx 1.4$ extends over a larger and larger range of scales, with the maximum size of the slip avalanches diverging like $\left(\tau_{\mathrm{c}}-\tau_{\mathrm{ext}}\right)^{-2}$. This behavior is almost identical with that observed in the 2D dislocation dynamics simulations reported in Section 
2 (Figure 3). Even the absolute avalanche sizes are similar if one uses the relation $\Delta \gamma=b L_{\text {tot }} / L_{\mathrm{s}}^{2}$ with $L_{\mathrm{s}}=128 \rho^{-1 / 2}$ to convert between data in Figures 4 and 3. This makes it plausible that both models, though conceptually different in their formulation, not only belong to the same universality class but describe the same physical reality. For completeness, the insert of Figure 4 shows also the cumulative distribution of avalanches occurring at any stress level; as predicted in the previous section, this scales with an exponent $\kappa+\sigma \approx 1.9$.

In addition to strain increments, the present continuum model because of his time-continuous dynamics allows to evaluate the strain rate evolution during an avalanche. This allows us to determine the distribution of peak strain rates $\dot{\gamma}_{\max }=: A$. Again, one observes a power law $p(A) \propto A^{-\kappa_{A}}$ with $\kappa_{A} \approx 2$. This is again in line with the expectation for mean-field depinning, and the exponent $\kappa_{A}=2$ is in good agreement with the exponents deduced from peak amplitude distribution of $\mathrm{AE}$ bursts in ice single crystals [15]. Finally, the avalanche durations are found to scale in proportion with the avalanche amplitudes.

Slip pattern and surface roughening Numerical simulation of the continuum model defined by Eqs. (3.3)-(3.4) yields strongly anisotropic, striated strain patterns (Figure 5) with strong correlations in the $x$ direction (the direction of the slip plane) but weak correlations in the normal direction. This can be readily understood by looking at the elastic interactions in Fourier space: The Fourier transform of the elastic kernel is zero along the $k_{x}$ and $k_{y}$ directions, see Eq. (3.2). While fluctuations along the $k_{x}$ direction are damped due to the second-order gradient term in Eq. (3.3), those along the $y$ direction are not. The model is, hence, capable of representing at least qualitatively the slip anisotropy which in real crystals is a direct consequence of the glide motion of dislocations on slip planes.

To compare with experimental observations of surface profiles, one may integrate the strain profile along the $y$ direction for some fixed $x=x_{0}$ to obtain a displacement profile along the corresponding plane normal to the slip direction:

$$
y(x)=\int_{0}^{x}[\gamma(x)-\langle\gamma\rangle] \mathrm{d} x .
$$
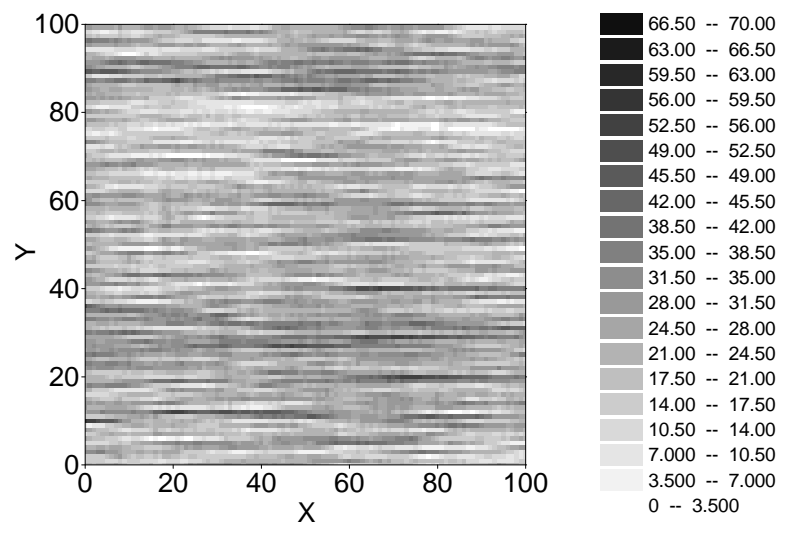

Figure 5: Strain pattern obtained after simulation of a system of size $256 \times 256$ to an average strain of $20 b \sqrt{\rho}$ (slip direction from left to right); greyscale: local strain in units of $b \sqrt{\rho}$ 


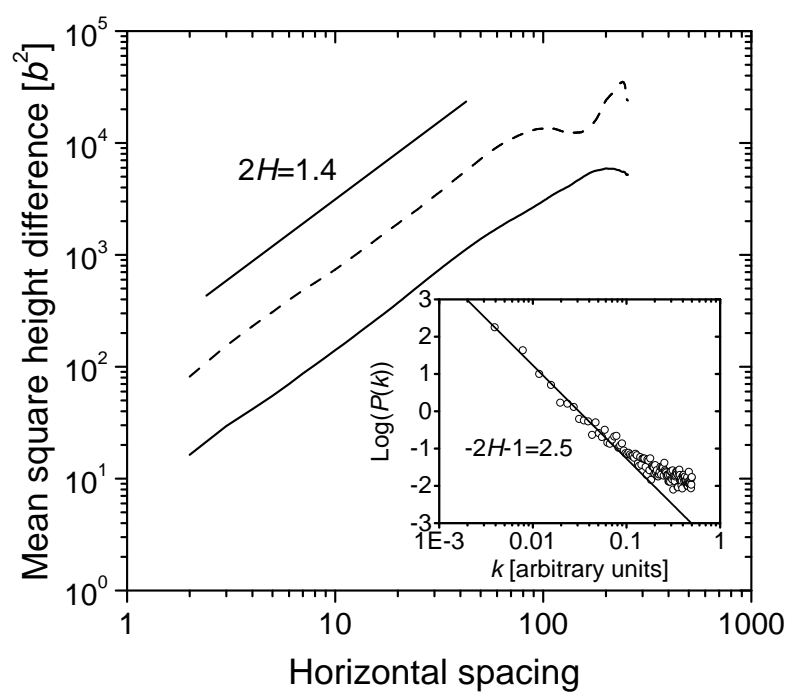

Figure 6: Mean square height difference vs. horizontal distance for surface profiles obtained from a system of size $512 \times 512$ after simulation to average strains of $6 b \sqrt{\rho}$ and $20 b \sqrt{\rho}$; each graph has been averaged over 10 simulated profiles; insert: Average power spectrum of the simulated surfaces for $\gamma=20 b \sqrt{\rho}$

Assuming that surface-specific effects can be neglected (near a free surface, the elastic interactions are modified by the surface boundary conditions which are not taken into account in our model), the morphology of such simulated 'surfaces' may be compared with that of real ones.

Analysis in terms of self-affine roughness was performed by studying the height-height correlation function and the power spectrum of the simulated surfaces [12]. The results can be summarized as follows (see Figure 6):

- The surfaces are self-affine up to a correlation length which is proportional to the size of the simulated system. They can be characterized by a strain-independent roughness exponent $\zeta \approx 0.7$.

- Increasing the total strain leads to growth of the profiles but does not change the roughness exponent or the correlation length (which is anyway determined by the system size). At large strains, the rms surface roughness on a given scale grows as the square root of strain.

- The numerical value of the roughness exponent compares well with the experimental observations, whereas the growth exponent of about 0.5 is slightly below the exponent of 0.8 observed during the single-slip deformation Stage I of $\mathrm{KCl}$ single crystals [4].

\section{Discussion and conclusions}

Our investigation shows that yielding can be modelled as a depinning transition. The continuum model discussed in Section 3 yields results that are in good agreement with experimental observations: The model predicts avalanche dynamics of plastic flow with an exponent $\kappa=1.4$ for the strain increments or, equivalently, the energy releases, whereas acoustic emission measurements 
indicate an exponent around 1.5 for the energy releases. The exponent of the cumulative distribution is predicted to be 1.9, again in good agreement with experiment. Finally, the predictions of a self-affine surface morphology with a roughness exponent $\zeta \approx 0.7$ agree well with experimental data obtained during the single-slip regime of the hardening curve of $\mathrm{KCl}$ single crystals [4]. The fact that one model covers observations made on quite different materials (Ice in case of the acoustic emission measurements, $\mathrm{Ni}$ and $\mathrm{NiAl}$ in case of the microcrystal experiments, $\mathrm{KCl}$ for the surface investigations) may indicate the universality that is commonly associated with critical behavior. However, it may be noted that all these observations were made in circumstances where deformation proceeds in single slip, and the situation may be different if multiple slip systems have to be considered (see also the systematic differences observed in polycrystalline samples, [15]). Since the strain rate acts as an order parameter of the 'yielding transition', multiple slip may qualitatively change the critical behavior: the number of independent components of the strain rate tensor defines the dimensionality of the order parameter, which together with the space dimensionality and interaction range defines the universality class of the transition; hence, single and multiple slip may be in different universality classes. Further investigations, both theoretical and experimental, are required to understand how the collective phenomena change in case of deformation on multiple slip systems.

The mean-field theory of depinning transitions can explain the observed avalanche statistics of the continuum model. However, it can by definition not tell anything about the spatial organization of slip. The formation of 'slip lines' as shown in Figure 5 hinges on the second-order gradient term in Eq. (3.3), which scales in Fourier space like $\vec{k}^{2} \gamma(\vec{k})$ and is, hence, on large scales irrelevant in comparison with the long range elastic term, Eq. (3.2). However, the second-order gradient term has a decisive influence on the small-scale morphology of the deformation patterns, as it breaks the symmetry existing in the kernel in Eq. 3.1 between the $x$ and $y$ directions, and suppresses deformation heterogeneities in the direction of dislocation glide. The model envisages surface roughening as a result of the stochastic superposition of many 'slip events' which have, however, long-range spatial correlations (for a more detailed discussion of this aspect, see [16].) The model correctly predicts the roughness exponent $\zeta=0.7$ observed in experiment, however, no theoretical explanation is presently available for this value.

Perhaps the most striking feature of our investigation is the near-complete agreement of the results deduced from two conceptually different models. In Section 2, we have the motion of discrete dislocations, described by an automaton model with deterministic evolution and stochastic initial conditions. In Section 3, by contrast, we have the time-continuous evolution of stress and strain fields, with randomness in the microstructure evolution being described in terms of a randomly fluctuating stress field. The agreement of both models indicates that there may exist a systematic coarse-graining procedure leading from the one to the other. This is another task to be accomplished in the future.

\section{Acknowledgement}

We gratefully acknowledge support of EPSRC under Grant No. GR/S20406/01. M.Z also acknowledges financial support of the Royal Society for attending SMPRI 2005. 


\section{References}

[1] M.-C. Miguel, A. Vespignani, S. Zapperi, J. Weiss and J.-R. Grasso, Intermittent dislocation flow in viscoplastic deformation, Nature 410 (2001) 667.

[2] J.Weiss and D.Marsan, Three-dimensional mapping of dislocation avalanches: clustering and space-time coupling, Science 299 (2003), 89.

[3] M. Zaiser, F. Madani, V. Koutsos and E.C. Aifantis, Self-affine suface morphology of plastically deformed metals, Phys. Rev. Letters 93 (2004) 195507.

[4] E. Nadgorny, J. Schwerdtfeger, F. Madani, V. Koutsos, E.C. Aifantis and M. Zaiser, Evolution of self-affine surface roughness in plastically deforming $\mathrm{KCl}$ single crystals, this volume.

[5] M.D. Uchic, D.M. Dimiduk, J. N. Florando and W. D. Nix, Sample dimensions influence strength and crystal plasticity, Science 305 (2004) 986.

[6] D.M. Dimiduk, M.D. Uchic and T.A. Parthasarathy, Size-affected slip behavior of pure Ni microcrystals, Acta Mater. 53 (2005) 4065.

[7] M. Zaiser, Statistical Modelling of Dislocation Systems, Materials Science and Engineering A, 309/310 (2001) 304.

[8] M.-C. Miguel, A. Vespignani, M. Zaiser and S. Zapperi, Dislocation Jamming and Andrade Creep, Phys. Rev. Letters 89 (2002) 165501.

[9] O. Perkovic, K. Dahmen and J.P. Sethna, Avalanches, Barkhausen noise, and plain old criticality, Phys. Rev. Lett. 75 (1995) 4528.

[10] R. LeSar, private communication.

[11] D.S. Fisher, Collective transport in random media: from superconductors to earthquakes, Physics Reports 301 (1998) 113.

[12] M. Zaiser and P. Moretti, Fluctuation phenomena in crystal plasticity - a continuum model, J. Stat. Mech. (2005) P08004.

[13] M. Zaiser and E.C. Aifantis, On the theory of gradient plasticity III: Random effects and slip avalanches, Int. Journ. Plasticity, in press.

[14] I. Groma, F. Csikor and M. Zaiser, Spatial correlations and higher-order gradient terms in a continuum description of dislocation dynamics, Acta Mater. 51 (2003) 1271.

[15] T. Richeton, J. Weiss and F. Louchet, Breakdown of avalanche critical behaviour in polycrystalline plasticity, Nature Materials 4, 465 (2005).

[16] M. Zaiser, Scale invariance in plastic flow of crystaline solids, Adv. Phys., submitted. 